\title{
Simultaneous monitoring of independent gene expression patterns in two types of cocultured fibroblasts with different color-emitting luciferases
}

\author{
Takako Noguchi ${ }^{1}$, Masaaki Ikeda ${ }^{2,3}$, Yoshihiro Ohmiya ${ }^{1,4}$ and \\ Yoshihiro Nakajima*1
}

Address: ${ }^{1}$ Cell Dynamics Research Group, Research Institute for Cell Engineering, National Institute of Advanced Industrial Science and Technology (AIST), 1-8-31 Midorigaoka, Ikeda, Osaka 563-8577, Japan, ${ }^{2}$ Molecular Clock Project, Research Center for Genomic Medicine, Saitama Medical School, Yamane, Hidaka, Saitama 350-1241, Japan, ${ }^{3}$ Department of Physiology, Saitama Medical School, Morohongo, Moroyama, Saitama 350-0495, Japan and ${ }^{4}$ Division of Molecular/Cell Imaging Department of Photobiology, Hokkaido University Graduate School of Medicine, West 7, North 15, Kita-ku, Sapporo 060-8638, Japan

Email:Takako Noguchi - t-noguchi@aist.go.jp; Masaaki Ikeda - mikeda@saitama-med.ac.jp; Yoshihiro Ohmiya - y-ohmiya@med.hokudai.ac.jp; Yoshihiro Nakajima* - y-nakajima@aist.go.jp

* Corresponding author

Published: 17 April 2008

BMC Biotechnology 2008, 8:40 doi:10.1 186/1472-6750-8-40
Received: II December 2007

Accepted: 17 April 2008

This article is available from: http://www.biomedcentral.com/I472-6750/8/40

(c) 2008 Noguchi et al; licensee BioMed Central Ltd.

This is an Open Access article distributed under the terms of the Creative Commons Attribution License (http://creativecommons.org/licenses/by/2.0), which permits unrestricted use, distribution, and reproduction in any medium, provided the original work is properly cited.

\begin{abstract}
Background: Luciferase assay systems enable the real-time monitoring of gene expression in living cells. We have developed a dual-color luciferase assay system in which the expression of multiple genes can be tracked simultaneously using green- and red-emitting beetle luciferases. We have applied the system to monitoring independent gene expressions in two types of cocultured fibroblasts in real time.

Results: Two Rat-I cell lines were established that stably express either green- or red-emitting luciferases under the control of the mBmall promoter, a canonical clock gene. We cocultured these cell lines, and gene expression profiles in both were monitored simultaneously. The circadian rhythms of these cell lines are independent, oscillating following their intrinsic circadian phases, even when cocultured. Furthermore, the independent rhythms were synchronized by medium change as an external stimulus.
\end{abstract}

Conclusion: Using this system, we successfully monitored independent gene expression patterns in two lines of cocultured fibroblasts.

\section{Background}

The broad application of luciferase reporter systems includes the transient assay of gene expression in cell extracts, the real-time monitoring of gene expression in living cells, and in vivo imaging of animals [1-6]. Luciferase reporter systems are widely used as sensitive, quantitative, and convenient measurement systems of gene expression in living cells [3]. The major advantages of luciferases over fluorescent reporters such as green fluorescent protein are that they do not require exogenous illumination because they catalyze luciferins to emit light directly, so the background emission from samples is extremely low $[4,5]$. The best-known luciferase is North American firefly (Photinus pyralis) luciferase. 
Dual-color luciferase assay systems have been developed that allow the simultaneous analysis of the expression of multiple genes [7-11]. These systems use two luciferases that emit either green or red bioluminescence with a common substrate, D-luciferin. Their emissions are then separated by an optical filter $[7,8,10,11]$. These systems enable the simultaneous monitoring of binary components. One of the applications of these systems is to measure the expression of two genes in homogeneous cell populations, an applications that has only just started being used [11]. Another application is the measurement of singlegene expression in heterogeneous cell populations, which is expected to serve the study of the cellular communications that are important in various biological processes such as development, differentiation and cancer growth [12-14]. However, to date, there has been no dual-color luciferase assay system applied to monitoring gene expression levels in cocultured heterogeneous cells.

In mammals, the central circadian pacemaker, located in the suprachiasmatic nucleus (SCN) of the hypothalamus, coordinates physiological rhythms such as the sleep-wake cycle, the rhythms of body temperature and the release of hormones [15]. Circadian oscillations in the expression of clock genes such as Bmal1, Per1, Per2, Cry1, and Cry2, are found not only in the SCN but also in peripheral tissues and in immortalized cells [15-18]. Individual neurons in the SCN work as independent oscillators. They communicate with each other and produce a synchronized rhythm [17]. In addition to SCN neurons, individual cultured fibroblasts (Rat-1 cells) or embryonic fibroblasts have been shown to function as self-sustained oscillators using single-cell imaging of bioluminescence or fluorescence $[18,19]$. Whereas the rhythms of cultured Rat-1 cells undergo rapid damping, they resume after stimuli such as changing the medium and the application of dexamethasone (DEX), a glucocorticoid analog [20]. Such damping of the rhythms may be caused by a loss of synchrony among cells $[18,19]$. These properties of Rat- 1 cells make them suitable as a model system to analyze gene expression patterns in cocultured cells.

To simultaneously study gene expression patterns in two cocultured types of cells, we have developed a real-time monitoring system of gene expression using the greenand red-emitting luciferases that we have previously reported [7]. The green-emitting luciferase was derived from a Japanese luminous beetle (Rhagophthalmus ohbai; $\lambda \max =550 \mathrm{~nm})$ and the red-emitting luciferase was derived from railroad worm (Phrixothrix hirtus; $\lambda \max =$ $630 \mathrm{~nm})[7,21,22]$. We established two types of stable Rat-1 cell lines that expressed either the green- or the redemitting luciferase under the control of the mBmal1 promoter. Here we demonstrate monitoring of the independent circadian oscillations of these two cell lines when cocultured and confirm the hypothesis that individual Rat-1 cells act as self-sustained oscillators. The results show that this dual-color luciferase assay system serves as an effective tool to investigate independent gene expression patterns in coculture systems.

\section{Results \\ Establishment of two stable cell lines reporting circadian rhythms using different color-emitting luciferases}

To allow observation of independent circadian rhythms in cocultured cells, we chose the mBmal1 promoter because mBmal1 gene expression shows a clear circadian rhythm in cultured fibroblasts [19].

Rat-1 cell lines stably expressing either the green- or the red-emitting luciferases under the control of the mBmal1 promoter were generated as described in the Methods. We refer to these cell lines as Bmal1-GR and Bmal1-RED, respectively. First, we measured the bioluminescence spectra of these cells, because the spectra are the most important factors for measuring each luciferase activity by splitting the emissions. The cells were trypsinized and harvested and the spectra were measured without destroying the cells. As shown in Figure 1, the bioluminescence spectra of both cell lines are almost the same as those from cellular extracts reported in a previous study [7],

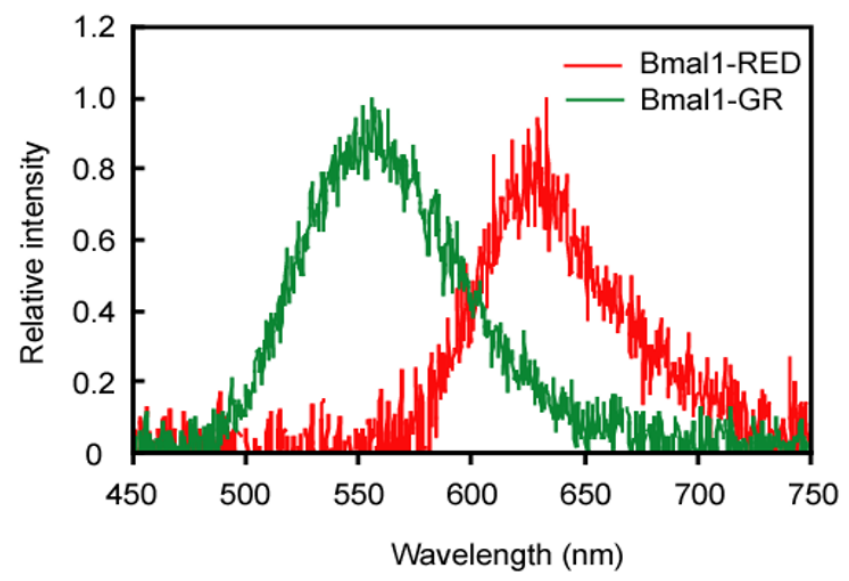

Figure I

Bioluminescence spectra of Bmal I-GR and

Bmal I-RED cell lines. Cells were incubated with 100 nM DEX for $18 \mathrm{~h}$ to synchronize circadian expression of the luciferases. Around the expected peak time of reporter gene expression, the Bmal I-GR ( $1.9 \times 10^{7}$ cells) and Bmal I-RED $\left(I .7 \times 10^{7}\right.$ cells) cells were trypsinized and collected from culture dishes. Cells were suspended in $250 \mu$ l of the luciferin medium. Their bioluminescence spectra were measured using an ABI 850 spectrophotometer (ATTO, Tokyo, Japan). The wavelengths of the light emitted (Bmall-GR, $\lambda$ max $=$ $556 \mathrm{~nm}$; Bmall-RED, $\lambda \max =633 \mathrm{~nm}$ ) were almost equal to those previously reported in cell extracts. 
demonstrating that each luciferase activity can be measured even when the cells are mixed. These cell lines showed clear circadian oscillations after treatment with DEX, which is known to synchronize circadian oscillation of clock genes in Rat-1 cells [20] (Fig. 2C, D). The circadian periods (mean \pm SEM) of Bmal1-GR \pm Bmal1-RED cells were $26.2 \pm 0.1 \mathrm{~h}(\mathrm{n}=8)$ and $23.7 \pm 0.1 \mathrm{~h}(\mathrm{n}=8)$, respectively.

\section{Real-time monitoring of gene expression in cocultured Bmall-GR and Bmal I-RED cells using the dual-color luciferase assay system}

If individual fibroblasts oscillate following their endogenous circadian periods, as shown previously $[18,19]$, then the circadian rhythms of cocultured Bmal1-GR and Bmal1-RED cells would be expected to keep their endogenous rhythms. We first attempted to demonstrate that independent circadian oscillations of two types of fibroblasts could be simultaneously monitored using different color-emitting luciferases. To do this, we triggered antiphasic oscillation in the two cell lines and examined whether rhythmic mBmal1 expression is affected by coculture.

Cell lines were grown separately in two wells formed by a silicon frame within a $35 \mathrm{~mm}$ culture dish (Fig. 2A, B). As controls, monocultures of either Bmal1-GR or Bmal1-RED cells were cultured in one of the two wells, and the other well was left empty (Fig. 2B, left and middle). The times of applying DEX to Bmal1-GR or Bmal1-RED cells were staggered by $12 \mathrm{~h}$. Thus, Bmal1-RED cells were stimulated with DEX first and the Bmal1-GR cells received DEX $12 \mathrm{~h}$ later. The same procedure was performed on the monocultures with blank cells adjacent. During the measurement of luciferase activity, the silicon frame was removed from the $35 \mathrm{~mm}$ dish so that the two cell lines could establish contact through the medium. The respective luciferase activities of both cell lines were measured simultaneously. The cocultured cell lines showed antiphasic circadian rhythms of each luciferase activity and the rhythm of each cell line was identical to that of the controls (Fig. 2C-E). Reflecting the longer endogenous circadian period of the Bmal1-GR cells, the peak time of Bmal1-GR cells was delayed progressively relative to that of the Bmal1-RED cells over 3 cycles (Fig. 2F, G). There were no significant differences in peak times or rates of damping between cocultures and monocultures $(P>0.05$, Mann-Whitney $U$ test; Fig. $2 \mathrm{~F}$, $G)$. Although it appears that the average rate of damping of Bmal1-GR cells was different from that of Bmal1-RED cells, the difference was not statistically significant. Thus, the varying gene expression levels in these two cocultured cell lines were monitored successfully.

\section{Analysis of the circadian phases of Bmal I-GR and Bmall-RED cells cocultured with direct contact}

We next examined whether signaling between neighboring cells plays a role in the synchronization of circadian rhythms. Taking advantage of the differences in circadian periods between the two types of Rat- 1 cell lines, we cocultured these cells with direct contact and examined rhythmic mBmall expression.

Equal numbers of Bmal1-GR and Bmal1-RED cells were mixed and grown in a dish. As controls, monocultures of either Bmal1-GR or Bmal1-RED cells were cultured individually (Fig. 3A, B). The cells were stimulated by replacing the culture medium with medium containing DEX. Both cell lines showed clear circadian rhythms in their luciferase activities, even when cocultured with direct contact (Fig. 3C). The peak times after the start of DEX treatment and the rate of damping in amplitude were analyzed statistically (Fig. 3D, E). The peak time of Bmal1-GR cells advanced to that of Bmal1-RED cells at peak 1 but it was reversed at peak 2 and the difference was enlarged at peak 3 . There were no significant differences in peak times or amplitude-damping rates between the monoculture and coculture systems $(P>0.05$, Mann-Whitney $U$ test $)$. Thus, the independence of the circadian rhythms of Rat-1 cells was confirmed using this dual-color luciferase assay system.

\section{Synchronization of phases by changing the medium}

To monitor the synchronization process of the fibroblasts, we changed the medium of the two cell lines oscillating in antiphase as described above, because fibroblasts are synchronized by external stimuli such as changing the medium, the application of DEX and temperature changes $[18,19,23,26]$.

The medium was replaced $3 \mathrm{~d}$ after the DEX treatment of Bmal1-RED cells. After changing the medium, the independently phased circadian rhythms of Bmal1-GR and Bmal1-RED cells were shifted to a new phase and the peaks become closer to each other (Fig. 4). These results are consistent with reports that fibroblast cycles are synchronized by environmental cues rather than a coupling among fibroblasts $[18,19,23]$. This result shows that the phase resetting of the two cell lines could be successfully and simultaneously monitored using the dual-color luciferase assay system.

\section{Discussion}

In this study, we report on a system for real-time monitoring of gene expression using green- and red-emitting luciferases, here used to investigate circadian rhythms in cocultured cells. There are various methods to analyze gene expression using multiple luciferases in combination, taking advantage of unique characteristics of the 
A
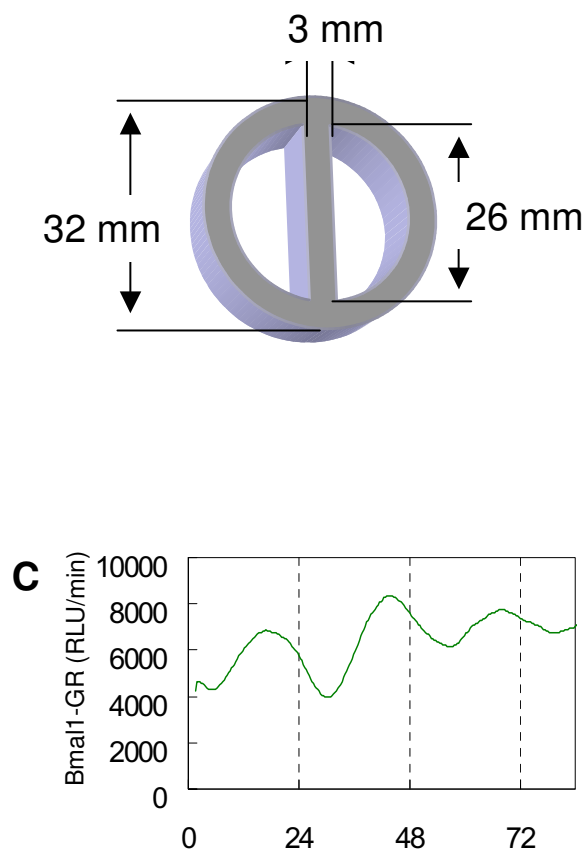

D

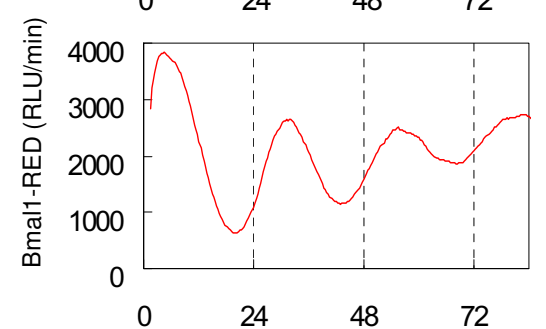

E

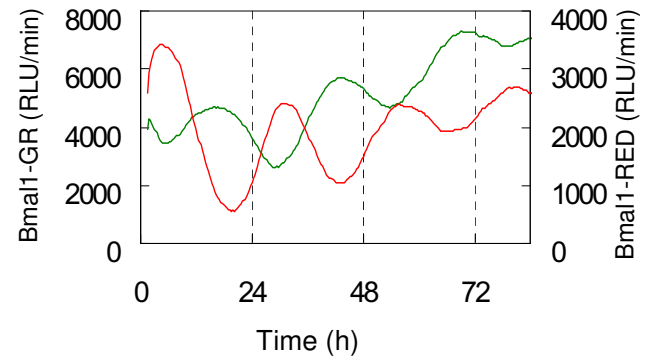

B

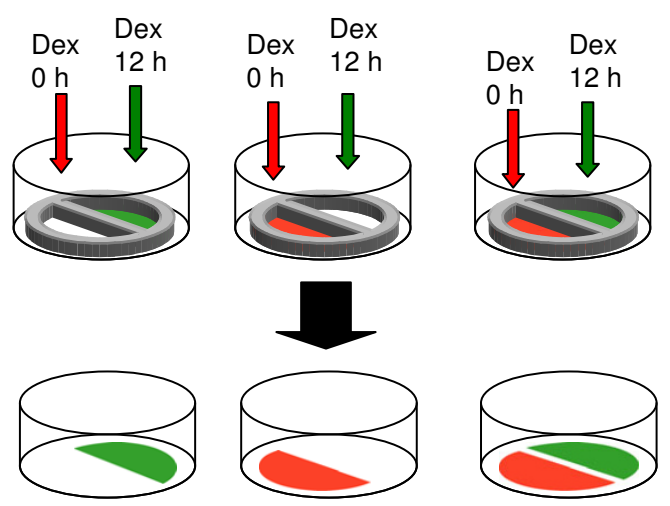

Time after DEX application

F to Bmal1-RED

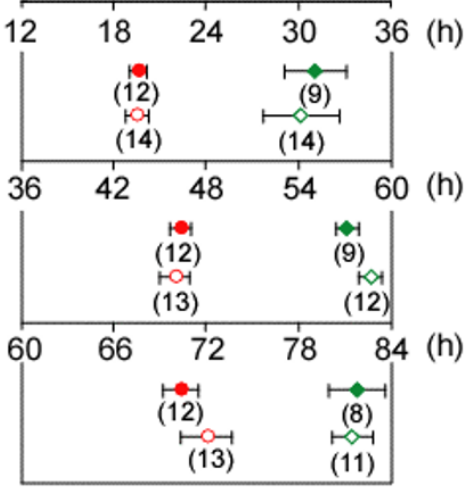

- Bmal1-GR/monoculture

- Bmal1-GR/coculture

- Bmal1-RED/monoculture

- Bmal1-RED/coculture

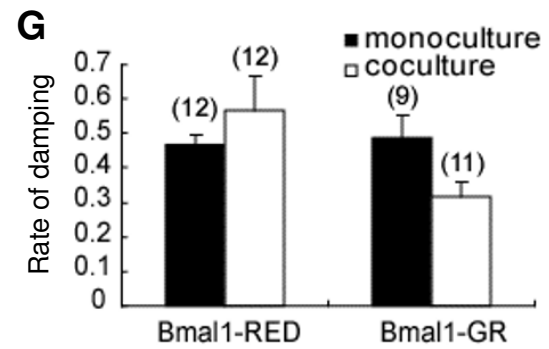

\section{Figure 2}

Real-time monitoring of gene expression in cocultured BmalI-GR and Bmal I-RED cells using the dual-color luciferase assay system. A: Schema of the silicon frame. Silicon frames of $32 \mathrm{~mm}$ major diameter and $26 \mathrm{~mm}$ inside diameter with a $3 \mathrm{~mm}$ wide divider were cut from a $3 \mathrm{~mm}$ thick silicon plate. B: Protocol for the induction of phases separated by $12 \mathrm{~h}$ in Bmal I-GR and Bmal I-RED cell lines. Bmal I-GR and Bmal I-RED cells were grown in separate wells of the silicon frame. Bmall-RED cells were treated with DEX medium (time $0 \mathrm{~h}$ ) and Bmal I-GR cells were treated I2 $\mathrm{h}$ later (time I2 h). After $2 \mathrm{~h}$, the medium in both wells was replaced with the luciferin medium (time $14 \mathrm{~h}$ ). The silicon frames were removed from the dishes and the bioluminescence was measured. Representative activities of green- and red-emitting luciferases are shown for Bmall-GR cells (C) and Bmall-RED cells (D) in monoculture, and in coculture (E). Luciferase activities are shown on the $Y$ axis and the measurement of time after starting bioluminescence is shown on the $\mathrm{X}$ axis. The independently phased circadian rhythms of luciferase activities from Bmall-GR and Bmal I-RED were monitored even when they were cocultured in the same dish. F: Average peak times are shown as the time after the start of DEX treatment of Bmall-RED cells. The symbols show the peak times of Bmal I-GR cells in monoculture (filled diamonds) and coculture (open diamonds); and Bmal I-RED cells in monoculture (filled circles) and in coculture (open circles). The number of samples is shown under the symbols. The bars show the SEM of the data. The phase of each cell line in coculture was identical to those in monoculture $(P>0.05$, Mann-Whitney $U$ test). G: Amplitude-damping rates of Bmal I-GR and Bmall-RED cells in monoculture and coculture are shown. There were no significant differences in damping rates between cell lines or between monocultures and cocultures $(P>0.05$, Mann-Whitney $U$ test). 


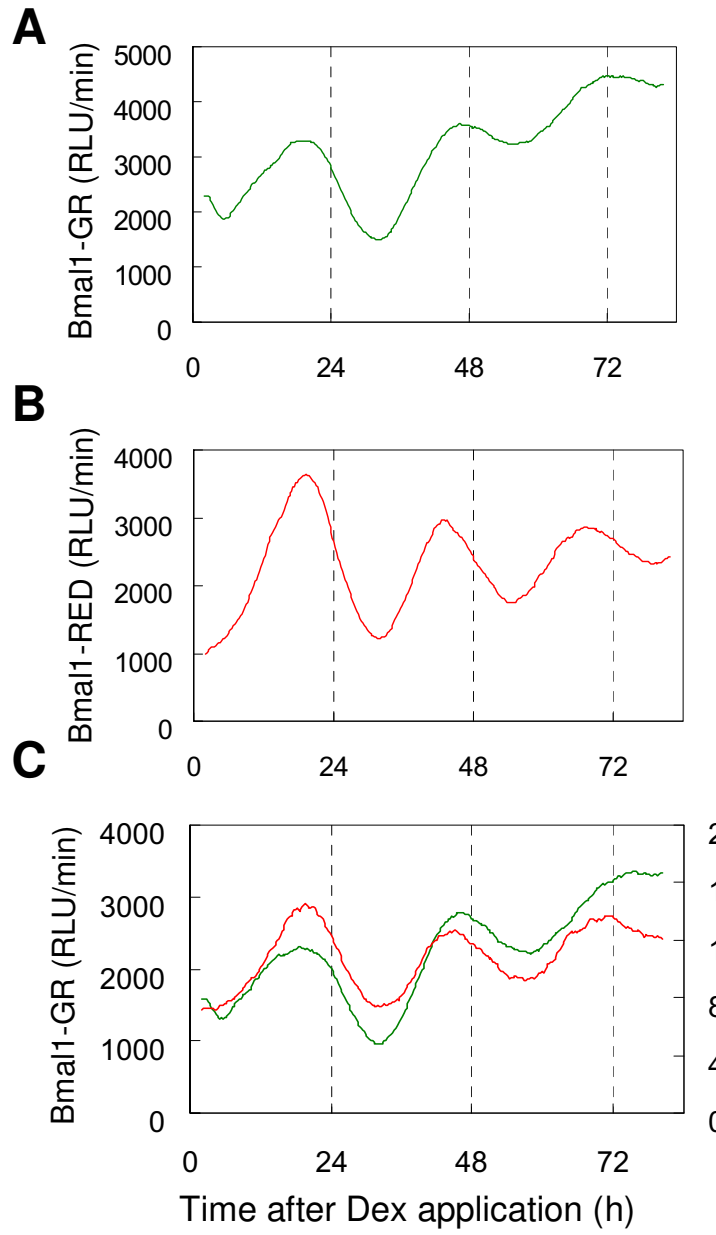

D Time after DEX application

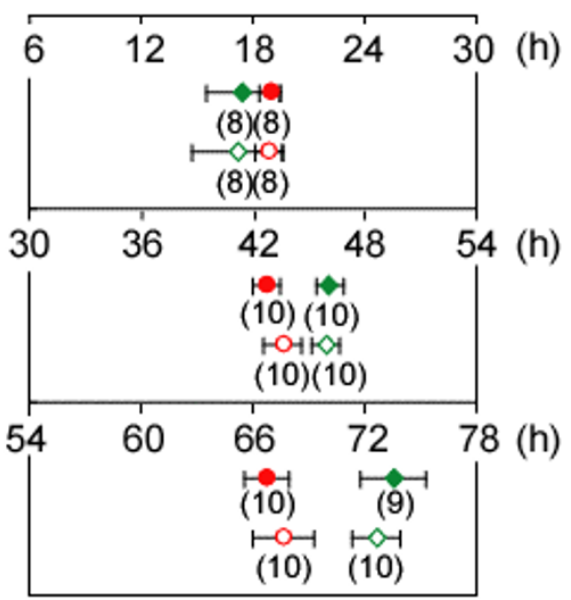

\section{- Bmal1-GR/monoculture - Bmal1-GR/coculture - Bmal1-RED/monoculture - Bmal1-RED/coculture}

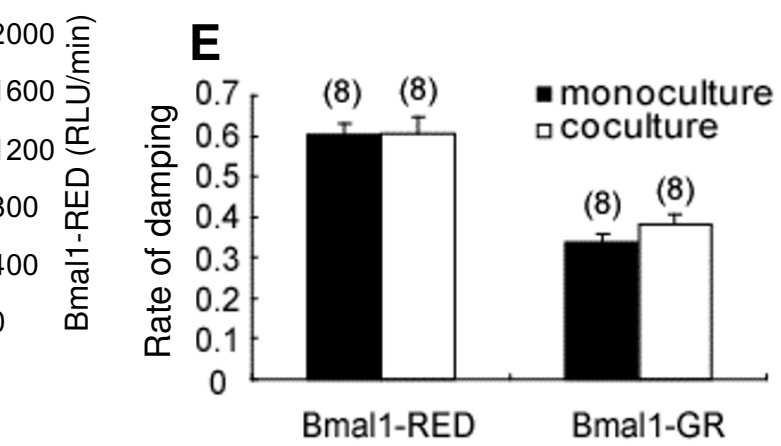

Figure 3

Analysis of the circadian phases of BmalI-GR and Bmal I-RED cells cocultured with direct contact. The bioluminescence activities of Bmal I-GR (A), Bmal I-RED (B), and cocultures of these cells (C) were measured. The luciferase activities are shown on the $Y$ axis and the time after starting measurement of the bioluminescence is shown on the $X$ axis. These cultures showed clear circadian rhythms. $D$ : Average peak times after the start of DEX treatment of BmalI-GR cells in monoculture (filled diamonds), in coculture (open diamonds), Bmal I-RED cells in monoculture (filled circles) and in coculture (open circles). The number of samples is shown under the symbols. The bars show the SEM of the data. The phases of each cell line gradually became separated in both cocultures and monocultures. E: Amplitude-damping rates of BmalI-GR and BmalI-RED cells in monoculture and coculture are shown. There were no significant differences in damping rates between cell lines or between monocultures and cocultures $(P>0.05$, Mann-Whitney $U$ test).

luciferases. Firefly and sea pansy (Renilla) luciferases are commonly used together to report expression levels of two different genes (e.g., the Dual-Luciferase ${ }^{\circledast}$ Reporter Assay System; Promega, Madison, WI, USA). The bioluminescence of these luciferases are counted separately, using two different substrates, D-luciferin and coelenterazine, required by each luciferase. The combination of sea-firefly (Vargula hilgendorfii) luciferase, a secreted protein, and firefly luciferase, a nonsecreted protein, has been successfully employed to report gene expression in mouse tissues [24].
Some other dual-color luciferase assay systems have now been reported [7-10]. The green- and red-emitting luciferases of these other systems were derived from the Jamaican click beetle [9], the North American firefly [10], and the railroad worm $[7,8,21]$. The luciferases used in this report were derived from the Japanese luminous beetle $[7,22]$ and the railroad worm $[7,8,21]$. The dual-color luciferase assay system has the following advantages: 1) the emission colors are separable by an optical filter, 2) they have a common luminescent substrate, and 3) the emission spectra are stable under physiological intracellular $\mathrm{pH}$ conditions $[7,8,21,22]$. The applications of dual- 


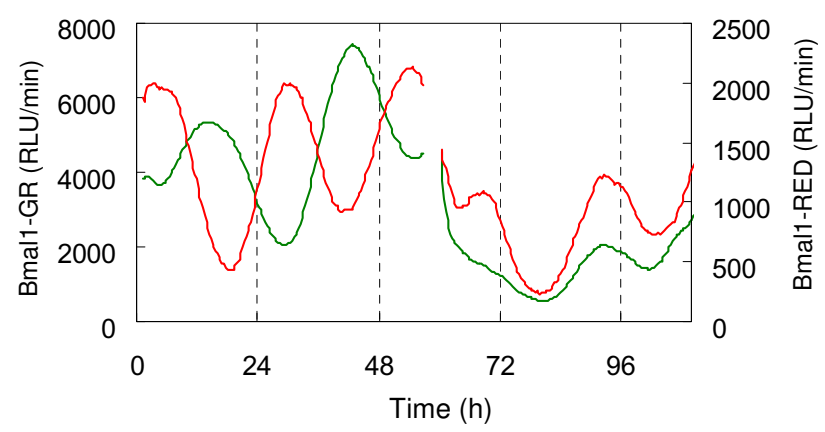

Figure 4

Synchronization of the phases by changing the medium. Antiphasic oscillations were induced in Bmall-GR and Bmall-RED cells, as shown in the figure. After $3 \mathrm{~d}$ of measurement, the medium was replaced with fresh luciferin medium. The independently phased circadian rhythms of the luciferase activities of Bmal I-GR and Bmal I-RED cells were reset by the medium change. Luciferase activities are shown on the $Y$ axis and the time after starting measurement of the bioluminescence is shown on the $X$ axis.

color luciferase systems have previously been limited to transient assays of gene expression in cell extracts, with the exception of one report of real-time monitoring of the expression of two genes in cyanobacteria [11]. In this report, we established real-time monitoring of gene expression in living mammalian cells. To demonstrate the further possible application of a dual-color luciferase assay system, we applied this system to monitoring gene expression patterns in two types of cocultured cell lines. In this system, furthermore, the orange-emitting luciferase can be employed as a third luciferase [7]. The third luciferase may serve to monitor the gene expression of a third cell line or to normalize the bioluminescence of the other two luciferases.

Here we demonstrated that the independent circadian oscillations of two types of cocultured stable cell lines could be reported by green- and red-emitting luciferases. These cell lines, which express green- and red-emitting luciferases under control of the mBmal1 promoter, showed about a $2.5 \mathrm{~h}$ difference in their periods. This may be caused by a positional effect arising from where the vectors are integrated in the genome rather than differences between the luciferases, as the periods of Rat-1 cells transiently transfected with these plasmids were not significantly different (data not shown). The diversity of periods among the individual fibroblasts from which these lines are derived may also cause the difference in periods $[18,19]-$. The separate luciferase activities of the cells in coculture were identical to those found in monocultured controls. We were also able to detect that the desynchronized circadian oscillations of stable lines became synchronized after changing the medium. In accordance with previous findings, our results confirm that these fibroblasts oscillate independently, following their individual triggered phases and intrinsic periods, and that they are transiently synchronized by external cues such as changing the medium $[19,20,23]$. However, we cannot rule out the possibility of coupling between fibroblasts because continuous medium replacement may be required for synchronizing factors to deliver the phase information for resetting cells.

The amplitudes of the circadian rhythms of fibroblasts cultured en masse undergo rapid damping, caused by a loss of synchrony $[18,19]$. Previous studies have investigated circadian communication between fibroblasts through mathematical modeling [18], fluorescent reporters [18] or luciferase reporters [25]. Using this dual-color luciferase assay system we could observe circadian rhythms with different phases and periods of both cell lines simultaneously. The existence of synchronizers of circadian rhythms is suggested for the SCN and other tissues because SCN and peripheral tissue explants are capable of self-sustained circadian oscillation for more than 20 cycles [26]. Coculture systems have contributed to the study of circadian communication among cells. Slices of SCN tissue induced long -lasting circadian rhythms in astrocytes [27], and immortalized SCN2.2 cells derived from the rat SCN imposed metabolic and Per1 gene expression in NIH3T3 cells [25]. Coculture systems using this dual-color luciferase assay system will thus help the search for circadian synchronizers in the body.

Cocultures of cells and tissues are used in many studies, for example, in studying signaling between glial cells and neurons, in examining the proliferation of cancer cells, and in observing cell differentiation [12-14]. This dualcolor luciferase assay system will contribute to the analysis of the interaction of cells in many biological fields. It will add further efficiency and versatility to the study of cellular communication by enabling the simultaneous monitoring of different gene expression patterns in cells in coculture.

\section{Conclusion}

Using this dual-color luciferase assay system, we were able to demonstrate independent circadian oscillations of mBmal1 expression in cocultured Rat-1 cell lines. This system is a simple and effective solution to the problems associated with conventional assays of cellular communication and can be used for any tissues or cells into which reporter gene constructs can be introduced. 


\section{Methods \\ Cell culture and generation of cell lines}

F2408 (Rat-1) cells (Health Science Research Resources Bank, Osaka, Japan) were grown in Dulbecco's modified Eagle's medium (DMEM; Sigma-Aldrich, St Louis, MO, USA) with $10 \%$ fetal bovine serum (FBS; ICN Biochemicals, Aurora, OH, USA), $0.1 \mathrm{mg} / \mathrm{ml}$ streptomycin (Nacalai Tesque, Kyoto, Japan), and $100 \mathrm{U} / \mathrm{ml}$ penicillin (Nacalai Tesque) at $37^{\circ} \mathrm{C}$ in a humidified $5 \% \mathrm{CO}_{2}$ incubator. Reporter plasmids, Bp/915-mGr and Bp/915-mRed, respectively expressing green- and red-emitting luciferases under the control of the mBmal1 promoter $(-816 /+99)$ $[28]$, were constructed as reported $[7,8]$. The green- and red-emitting luciferases are commercially available as SLG and SLR (MultiReporter Assay System, Tripluc ${ }^{\circledast}$, Toyobo, Osaka, Japan). For stable transfection, Rat-1 cells were cotransfected with the plasmid and pSV-Neo as the expression vector for the neomycin resistance gene using Lipofectamine 2000 (Invitrogen, Carlsbad, CA, USA) according to the manufacturer's instructions. After two days of transfection, the cells were subcultured for selection with $500 \mu \mathrm{g} / \mathrm{ml}$ geneticin (G418; Nacalai Tesque, Kyoto, Japan). We named the subcultured G418-resistant cell line with Bp/915-mGr as Bmal1-GR and the cell line with Bp/915-mRed as Bmal1-RED.

\section{Measurement of bioluminescence spectra}

Bmal1-GR and Bmal1-RED were grown to confluence in $10 \mathrm{~cm}$ dishes. The circadian rhythms were synchronized by replacing the culture medium with DEX medium consisting of DMEM without phenol red (Gibco BRL, Gaithersburg, MD, USA) plus 10\% FBS and 100 nM DEX (Nacalai Tesque). The cells were trypsinized and harvested from dishes after $18 \mathrm{~h}$. Cells were centrifuged and suspended in $250 \mu \mathrm{l}$ of luciferin medium consisting of DMEM without phenol red (Gibco), 10\% FBS, $100 \mathrm{nM}$ DEX, and $200 \mu \mathrm{M}$ D-luciferin (Toyobo, Osaka, Japan). Bioluminescence spectra were measured using an AB1850 spectrophotometer (ATTO, Tokyo, Japan) without destroying the cells.

\section{Culture of stable cell lines}

Bmal1-GR and Bmal1-RED cells were grown in DMEM supplemented with $200 \mu \mathrm{g} / \mathrm{ml}$ G418. To culture two types of Rat-1 cells in separate wells, silicon frames with a 32 $\mathrm{mm}$ major diameter and a $26 \mathrm{~mm}$ inside diameter with a $3 \mathrm{~mm}$ wide divider were cut from a 3 -mm-thick silicon rubber plate (Togawa Rubber, Osaka, Japan) (Fig. 3A). The silicon frames were soaked in $70 \%$ ethanol (Nacalai Tesque, Kyoto, Japan) and placed on $35 \mathrm{~mm}$ dishes. After the ethanol was dried up at room temperature, the frame became stuck tightly to the bottom of a $35 \mathrm{~mm}$ dish. Bmal1-GR $\left(2 \times 10^{5}\right)$ cells and Bmal1-RED $\left(2.4 \times 10^{5}\right)$ cells were seeded in separate wells of the silicon frame. After 2-3 d, the culture medium from the Bmal1-RED cells grown to $100 \%$ confluence was replaced with DEX medium (time $0 \mathrm{~h}$ ). Bmal1-GR cells were treated with DEX medium $12 \mathrm{~h}$ later (time $12 \mathrm{~h}$ ). After $2 \mathrm{~h}$ of DEX treatment of the Bmal1-GR cells, the medium in both wells was replaced with luciferin medium (time $14 \mathrm{~h}$ ). The silicon frame was removed from the $35 \mathrm{~mm}$ dish so that the two cell lines were in contact through the luciferin medium. The luciferin medium was covered with $2 \mathrm{ml}$ mineral oil (Sigma) to prevent evaporation.

To culture Bmal1-GR and Bmal1-RED cells with direct contact, the two lines were mixed and seeded in $35 \mathrm{~mm}$ dishes at a density of $3 \times 10^{5}$ cells each. After $2-3 \mathrm{~d}$, the cells at confluence were treated with DEX medium. After $2 \mathrm{~h}$ of DEX treatment, the medium was replaced with luciferin medium and covered by $2 \mathrm{ml}$ of mineral oil.

\section{Real-time monitoring by dual-color luciferase assay system} The real-time monitoring of $\mathrm{mBmal} 1$ expression using green- and red-emitting luciferases was performed with a dish-type luminometer (AB2500 Kronos, ATTO), according to a previous report, with slight modification $[7,8]$. The luminometers were placed in an incubator under $5 \%$ $\mathrm{CO}_{2}$ in air at $20^{\circ} \mathrm{C}$. The cultures were incubated at $37^{\circ} \mathrm{C}$ in the luminometers and bioluminescences were monitored for $1 \mathrm{~min}$ at $10-20 \mathrm{~min}$ intervals in the absence or presence of a $620 \mathrm{~nm}$ long-pass filter (R62 filter, Hoya, Tokyo, Japan) $[7,8]$. The bioluminescence count obtained was expressed in relative light units (RLU). Each greenand red-emitting luciferase activity was calculated from the total RLU (F0) and the RLU passed through the R62 filter (F1), as reported [7]. Briefly, the light intensity of green-emitting luciferase $(\mathrm{G})$ and red-emitting luciferase (R) were obtained from F0 and F1 and from the optical filter's transmission coefficients for the green- $\left(\kappa_{\mathrm{G}}\right)$ and the red-emitting $\left(\kappa_{\mathrm{R}}\right)$ luciferases using the following simultaneous equation.

$$
\left(\begin{array}{l}
F 0 \\
F 1
\end{array}\right)=\left(\begin{array}{ll}
1.0 & 1.0 \\
\kappa_{G} & \kappa_{R}
\end{array}\right)\left(\begin{array}{l}
G \\
R
\end{array}\right)
$$

\section{Analysis of circadian rhythms in bioluminescence}

To determine the periods, we analyzed the data over $4 \mathrm{~d}$ by subtracting a $24 \mathrm{~h}$ moving average from the crude data and obtained a best-fit cosine curve for the data using a least squares spectrum method [29]. To determine the peak times and amplitude-damping rate, serial records of bioluminescence were smoothed using a 10-point (2-3 h) moving average [15]. The circadian peaks and troughs were calculated as the highest and lowest points of the smoothed data. We determined peaks 1, 2, and 3 as the highest points that appeared $10.0-25.0 \mathrm{~h}, 40.0-55.0 \mathrm{~h}$, and 60.0-80.0 $\mathrm{h}$ after the start of DEX treatment, respectively, and troughs 1, 2, and 3 as the lowest points following peaks 1, 2, and 3, respectively. The RLU difference 
between peak 1 and trough 1 , and peak 2 and trough 2 were determined as amplitude 1 and amplitude 2, respectively. The ratio of amplitude 2 to amplitude 1 was determined as the amplitude-damping rate.

\section{Authors' contributions}

TN carried out the experiments on measuring bioluminescence, designed experiments, analyzed data, and drafted the manuscript. MI cloned mBmal1 and its critical promoter region. YO initiated the project and helped in writing the manuscript. YN constructed cell lines, established the dual-color luciferase assay system, and supervised the experiments. All authors read and approved the final manuscript.

\section{Acknowledgements}

We thank Ms N. Ueda and Ms A. Oka of AIST for technical assistance; Mr. H. Kubota, Mr. A. Asakawa and Mr. T. Enomoto of ATTO Co. for the development of instruments; Dr. K. Watanabe of Dokkyo University School of Medicine for offering a data analysis program; and Dr. S. Honma and Dr. K. Honma of Hokkaido University for helpful discussions and suggestions. This work was supported by a NEDO grant (Dynamic Biology Project) from the Ministry of Economy, Trade, and Industry of Japan and by a Grant-in-Aid for Scientific Research (I8790 I75) from the Ministry of Education, Culture, Sports, Science, and Technology, Japan.

\section{References}

I. Seliger HH, McElroy WD: The colors of firefly bioluminescence: enzyme configuration and species specificity. Proc Natl Acad Sci USA 1964, 52:75-8I.

2. Bronstein I, Fortin J, Stanley PE, Stewart GS, Kricka LJ: Chemiluminescent and bioluminescent reporter gene assays. Anal Biochem 1994, 219:169-181.

3. Naylor L: Reporter gene technology: the future looks bright Biochem Pharmacol 1999, 58:749-757.

4. Welsh DK, Imaizumi T, Kay SA: Real-time reporting of circadianregulated gene expression by luciferase imaging in plants and mammalian cells. Methods Enzymol 2005, 393:269-288.

5. Gross S, Piwnica-Worms D: Spying on cancer: molecular imaging in vivo with genetically encoded reporters. Cancer Cell 2005, 7:5-15

6. Kondo T, Strayer CA, Kulkarni RD, Taylor W, Ishiura M, Golden SS, Johnson $\mathrm{CH}$ : Circadian rhythms in prokaryotes: luciferase as a reporter of circadian gene expression in cyanobacteria. Proc Natl Acad Sci USA 1993, 90:5672-5676.

7. Nakajima $Y$, Kimura T, Sugata $K$, Enomoto $T$, Asakawa A, Kubota $H$, Ikeda M, Ohmiya $Y$ : Multicolor luciferase assay system: onestep monitoring of multiple gene expressions with a single substrate. Biotechniques 2005, 38:891-894.

8. Nakajima $Y$, Ikeda M, Kimura T, Honma S, Ohmiya Y, Honma K, Shuman S: Bidirectional role of orphan nuclear receptor $\mathbf{R O R} \alpha$ in clock gene transcriptions demonstrated by a novel reporter assay system. FEBS Lett 2004, 565:122-126.

9. Ogura R, Matsuo N, Wako N, Tanaka T, Ono S, Hiratsuka K: Multicolor luciferases as reporters for monitoring transient gene expression in higher plants. Plant Biotechnol 2005, 22:151-155.

10. Branchini BR, Southworth TL, Khattak NF, Michelini E, Roda A: Redand green-emitting firefly luciferase mutants for bioluminescent reporter applications. Anal Biochem 2005, 345: I 40-I 48.

II. Kitayama $Y$, Kondo T, Nakahira Y, Nishimura H, Ohmiya Y, Oyama $\mathrm{T}$ : An in vivo dual-reporter system of cyanobacteria using two railroad-worm luciferases with different color emissions. Plant Cell Physiol 2004, 45: 109-I I3.

12. Nueda ML, Baladron V, Sanchez-Solana B, Ballesteros MA, Laborda J: The EGF-like protein dlkI inhibits Notch signaling and potentiates adipogenesis of mesenchymal cells. J Mol Biol 2007, 367:|28|-|293.
13. Lie DC, Colamarino SA, Song HJ, Desire L, Mira H, Consiglio A, Lein ES, Jessberger S, Lansford H, Dearie AR, Gage FH: Wnt signalling regulates adult hippocampal neurogenesis. Nature 2005 , 437:1370-I375

14. Su G, Blaine SA, Qiao D, Friedl A: Shedding of syndecan-I by stromal fibroblasts stimulates human breast cancer cell proliferation via FGF2 activation. I Biol Chem 2007, 282: 14906-149|5.

15. Hastings M, O'Neill JS, Maywood ES: Circadian clocks: regulators of endocrine and metabolic rhythms. J Endocrinol 2007, 195:187-198.

16. Ko CH, Takahashi JS: Molecular components of the mammalian circadian clock. Hum Mol Genet 2006, I 5:R27I-R277.

17. Welsh DK, Logothetis DE, Meister M, Reppert SM: Individual neurons dissociated from rat suprachiasmatic nucleus express independently phased circadian firing rhythms. Neuron 1995 , 14:697-706.

18. Nagoshi E, Saini C, Bauer C, Laroche T, Naef F, Schibler U: Circadian gene expression in individual fibroblasts: cell-autonomous and self-sustained oscillators pass time to daughter cells. Cell 2004, I I 9:693-705.

19. Welsh DK, Yoo SH, Liu AC, Takahashi JS, Kay SA: Bioluminescence imaging of individual fibroblasts reveals persistent, independently phased circadian rhythms of clock gene expression. Curr Biol 2004, 14:2289-2295.

20. Balsalobre A, Brown SA, Marcacci L, Tronche F, Kellendonk C, Reichardt HM, Schutz G, Schibler U: Resetting of circadian time in peripheral tissues by glucocorticoid signaling. Science 2000 , 289:2344-2347.

21. Viviani VR, Bechara EJ, Ohmiya Y: Cloning, sequence analysis and expression of active Phrixothrix railroad-worms luciferases: relationship between bioluminescence spectra and primary structures. Biochemistry 1999, 38:827I-8279.

22. Ohmiya Y, Sumiya M, Viviani VR, Ohba N: Comparative aspects of a luciferase molecule from the Japanese luminous beetle, Rhagophthalmus ohbai. Sci Rept Yokosuka City Mus 2000, 47:3 I-38.

23. Hirota T, Okano T, Kokame K, Shirotani-lkejima H, Miyata T, Fukada Y: Glucose down-regulates Perl and Per2 mRNA levels and induces circadian gene expression in cultured Rat-I fibroblasts. J Biol Chem 2002, 277:44244-4425I.

24. Nishide SY, Honma S, Nakajima Y, Ikeda M, Baba K, Ohmiya Y, Honma K: New reporter system for Perl and Bmall expressions revealed self-sustained circadian rhythms in peripheral tissues. Genes Cells 2006, I I:II73-I I82.

25. Allen G, Rappe J, Earnest DJ, Cassone VM: Oscillating on borrowed time: diffusible signals from immortalized suprachiasmatic nucleus cells regulate circadian rhythmicity in cultured fibroblasts. J Neurosci 200I, 21 :7937-7943.

26. Yoo SH, Yamazaki S, Lowrey PL, Shimomura K, $\mathrm{Ko} \mathrm{CH}$, Buhr ED, Siepka SM, Hong HK, Oh WJ, Yoo OJ, Menaker M, Takahashi JS: PERIOD2::LUCIFERASE real-time reporting of circadian dynamics reveals persistent circadian oscillations in mouse peripheral tissues. Proc Natl Acad Sci USA 2004, 1 01:5339-5346.

27. Prolo LM, Takahashi JS, Herzog ED: Circadian rhythm generation and entrainment in astrocytes. J Neurosci 2005, 25:404-408.

28. Yu W, Nomura M, lkeda M: Interactivating feedback loops within the mammalian clock: BMALI is negatively autoregulated and upregulated by CRYI, CRY2 and PER2. Biochem Biophys Res Commun 2002, 290:933-94I.

29. Watanabe K, Katagai T, Ishida N, Yamaoka S: Anisomycin induces phase shifts of circadian pacemaker in primary cultures of rat suprachiasmatic nucleus. Brain Res 1995, 684: 179-184. 